JMB APB

\title{
Strong Bending of Purple Membranes in the M-state
}

\section{Dietmar Porschke*}

\author{
Max Planck Institut für \\ biophysikalische Chemie, Am \\ Fassberg 11, 37077 Göttingen \\ Germany
}

\begin{abstract}
Structure changes of purple membranes during the photocycle were analysed in solution by measurements of the electric dichroism. The D96Nmutant was used to characterize the M-state at neutral $\mathrm{pH}$. The transition from the resting state to $61 \%$ photo-stationary M-state is associated with a strong reduction of the dichroism decay time constant by a factor of $\sim 2$. Because the change of the time constant is independent of the bacteriorhodopsin concentration, the effect is not attributed to light-induced dissociation but to light-induced bending of purple membranes. After termination of light-activation the dichroism decay of the resting state is restored with a time constant close to that of the M-state decay, which is more than two orders of magnitude slower than proton transfer to the bulk. Thus, bending is not due to asymmetric protonation but to the structure of the M-state. A very similar reduction of decay time constants at a corresponding degree of light-activation was found for wild-type bacteriorhodopsin at $\mathrm{pH}$-values 7.8-9.3, where the lifetime of the M-state is extended. Light-induced bending is also reflected in changes of the stationary dichroism, whereas the overall permanent dipole moment remains almost constant, suggesting compensation of changes in molecular and global contributions. Bead model simulations indicate that disks of $\sim 1 \mu \mathrm{m}$ diameter are bent at a degree of photo-activation of $61 \%$ to a radius of $\sim 0.25 \mu \mathrm{m}$, assuming a cylindrical bending modus. The large light-induced bending effect is consistent with light-induced opening of the protein on the cytoplasmic side of the membrane detected by electron crystallography, which is amplified due to coupling of monomers in the membrane. Bending may function as a mechanical signal.
\end{abstract}

(c) 2003 Elsevier Ltd. All rights reserved.

Keywords: electric dichroism; rotational diffusion; bead model simulation; D96N; dipole moment

\section{Introduction}

Proton pumping during the photocycle of bacteriorhodopsin has been analysed actively over many years by virtually all available scientific techniques. ${ }^{1-4} \mathrm{~A}$ major part of research activity was focussed on studies of light-induced changes of local structure indicated by various spectro-

Abbreviations used: wt, wild-type; $\xi$, electric dichroism $\left(\xi=\left(\Delta A_{\|}-\Delta A_{\perp}\right) / \bar{A} ; \Delta A_{\|}\right.$and $\Delta A_{\perp}$, absorbance changes at parallel and perpendicular orientation of the polarizer to the field, respectively, $\bar{A}$, isotropic absorbance); index, $\infty$, limiting value of the dichroism at complete orientation; indices, AC and DC, data from measurements in AC-fields and DC-fields, respectively; index, $*$, light-activated state; e.g. $\xi_{\infty}^{\mathrm{DC} *}$, limiting dichroism from DC-measurements for lightactivated state.

E-mail address of the corresponding author: dpoersc@gwdg.de scopic parameters. Changes on the level of the global structure hardly received any attention. Probably this is partly due to the fact that it is more difficult to detect such changes at a sufficiently high sensitivity. The structure of bacteriorhodopsin has also been characterized by X-ray crystallography, electron crystallography and NMR analysis in its resting state and in different light-activated states. ${ }^{4-6}$ These studies provide a wealth of stereo-chemical information with many details on the protein structure. However, there is very little information on changes of global structure during the photocycle. This may be partly due to the fact that changes of global structure in the crystalline state are restricted due to packing.

In the present investigation the structure of purple membranes is analysed in solution where restrictions with respect to global changes do not exist. The analysis is based on molecular electro-optics, a set of techniques including electric 
dichroism and electric birefringence with a particularly high sensitivity for analysis of both local and global changes of macromolecular structures. ${ }^{7,8}$ Because of its high optical and electrical anisotropy, bacteriorhodopsin has been studied by these techniques in many different laboratories. ${ }^{9-19}$ However, electro-optical techniques have not been used yet to characterize different functional states of bacteriorhodopsin. Among the reaction states of bacteriorhodopsin the M-state is of particularly high interest, because this state is known to be critical for the pumping activity. The experimental data show a large change of the global structure during the M-state, indicating strong bending of the disks. Changes in bending of wt-bacteriorhodopsin disks induced by light were reported by Czege \& Rheinisch ${ }^{20,21}$ based on measurements of light-scattering intensities. However, as described in the Discussion, the essential results are completely different. The difference appears to be mainly due to the much higher sensitivity of the dichroism decay, reporting changes of global structure with a dependence upon the third power of the hydrodynamic dimensions. The molecular basis of the large bending effects seems to be changes of the protein structure occurring on the local level, which are amplified due to tight coupling of monomers up to large changes on the global level. Although a biological role of the bending effect is not known, the magnitude of the effect suggests a function as a simple mechanical signal.

\section{Results}

\section{Large change of electric dichroism and of rotational diffusion induced by light}

Analysis of reaction states during the photocycle by electro-optical procedures requires controlled application of light and electric field pulses. A special instrument has been constructed for this purpose (cf. Figure 1). Experiments for characterization of the photo-activated state were initiated by a light pulse. Then an electric field pulse followed, with a delay of $\sim 100 \mathrm{~ms}$. In most cases the light pulse was maintained until the end of the dichroism decay. Thus, dichroism transients were usually recorded in the photo-stationary state.

Characterization of reaction states generated during the photocycle requires a high degree of photo-activation, which is associated with very large changes of the absorbance at wavelengths around $570 \mathrm{~nm}$. Because these changes perturb the dichroism transients, the present measurements were mainly conducted at the wavelength $\lambda=458 \mathrm{~nm}$, where absorbance changes resulting from photoreactions of bacteriorhodopsin are minimal.

As shown by the example in Figure 2, the accuracy of dichroism transients measured at this wavelength is sufficiently high. Irradiation of light at a high intensity in the range $\lambda>500 \mathrm{~nm}$ induces a considerable change of the dichroism: the amplitude decreases by a factor of more than 2 and the dichroism decay is accelerated by a factor of $\sim 2$. An accurate description of the decay curves obtained for the D96N sample requires two exponentials both for the resting and the light-activated state. The time constants for the light-activated state $\left(\tau_{1}^{*}=7.2 \mathrm{~ms}, \tau_{2}^{*}=21.9 \mathrm{~ms}\right.$ with a relative amplitude for the second exponential $A_{2}^{*}=64.4 \%$ ) are clearly smaller than those in the resting state $\left(\tau_{1}=10.6 \mathrm{~ms}, \tau_{2}=49.0 \mathrm{~ms}, A_{2}=66.3 \%\right)$. The time constants of the dichroism decay are a very sensitive measure of the hydrodynamic dimensions. Thus, the change of the time constants indicates a strong decrease in the hydrodynamic dimensions upon light-activation. Furthermore, the observation of two exponentials indicates the existence of a distribution of particle sizes in the D96N sample.

\section{Contributions from light-scattering reflected by magic angle data}

When the light used for measurements is polarized to the magic angle $\left(55^{\circ}\right.$ with respect to the field vector), the electric dichroism is suppressed completely, whereas effects due to conformation changes and other reactions are not suppressed at all. ${ }^{8,22}$ A detailed analysis of the transients obtained at $\lambda=458 \mathrm{~nm}$ revealed a special contribution at this magic angle. In the wavelength range around $570 \mathrm{~nm}$, field-induced changes of the absorbance at the magic angle are negligible compared to the large dichroism amplitudes, whereas at $458 \mathrm{~nm}$ contributions at the magic angle cannot be neglected. An analysis of the magic angle amplitude $\Delta A_{55}$ measured for a given solution and with constant electric field pulses but different wavelengths shows that $\Delta A_{55}$ increases linearly with $1 / \lambda^{4}$, indicating that this amplitude results from lightscattering.

The effects observed at the magic angle are dependent on the concentration of bacteriorhodopsin. At high concentrations, e.g. in the range of $10 \mu \mathrm{M}$ and above, the field-induced transients are clearly affected by a reaction associated with a particularly slow rise and a relatively fast decay. The example in Figure 3 shows the slow rise of the transient recorded at the magic angle $\left(\vartheta=55^{\circ}\right)$ and an unusual shape of the transient recorded at parallel orientation of polarization plane and field vector $\left(\vartheta=0^{\circ}\right)$. The unusual shape at $\vartheta=0^{\circ}$ is due to superposition of a standard orientation effect and a "magic angle" effect. Thus, subtraction of the magic angle effect measured at $\vartheta=55^{\circ}$ from that measured at $\vartheta=0^{\circ}$ should reveal the unperturbed form of the orientation effect, as verified in Figure 3 . The validity of the correction is also indicated by the fact that the ratio $\Delta A_{0} / \Delta A_{90}$ of the stationary absorbance changes measured at $\vartheta=0^{\circ}$ and $\vartheta=90^{\circ}$ is far from the expected value ${ }^{7,8,22}-2$ before correction but in agreement with -2 after correction (within experimental accuracy $\pm 20 \%$ ). 


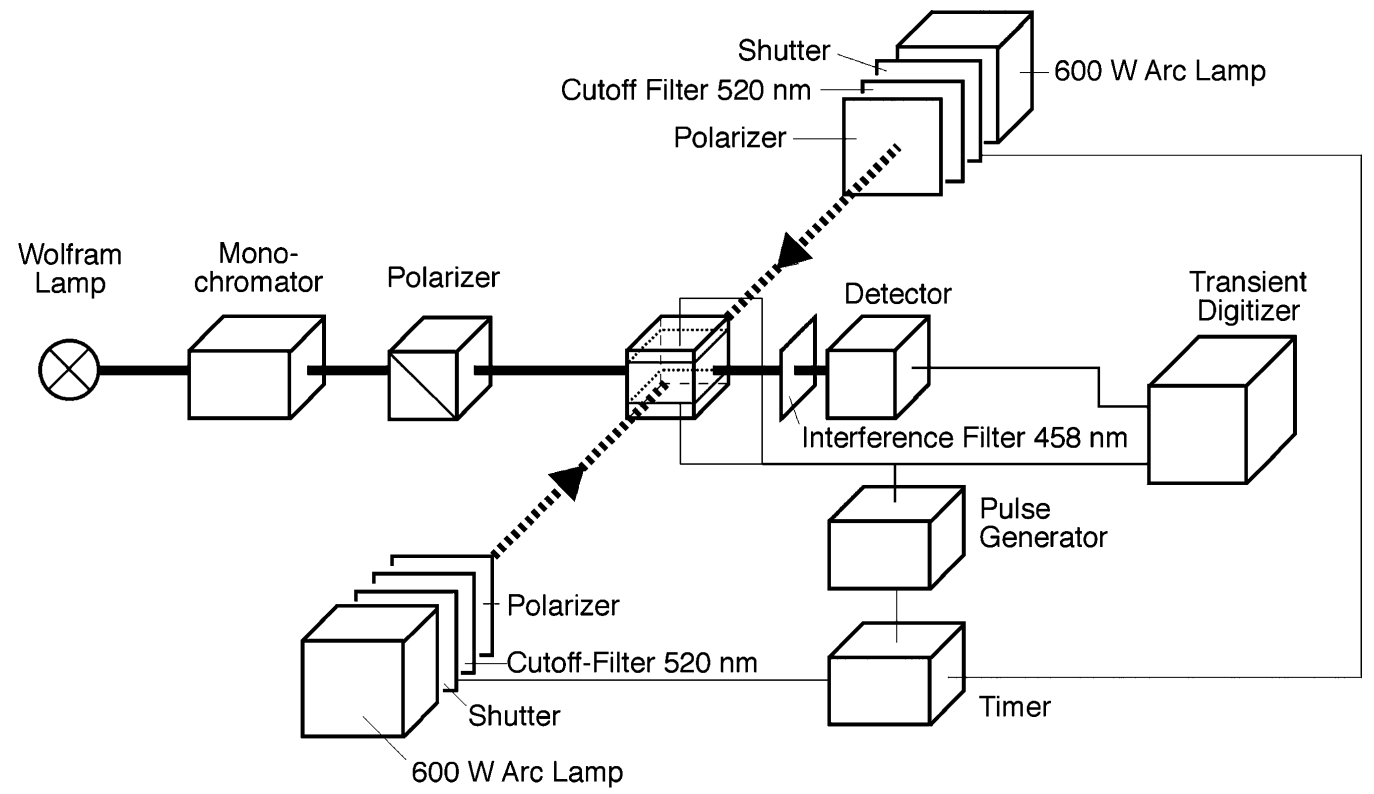

Figure 1. Scheme of the instrument used for the present measurements. The light pulses for photo-activation were generated by the two $600 \mathrm{~W}$ arc lamps. The light for measurements was generated by a $100 \mathrm{~W}$ wolfram halogen lamp. Light pulses and electric field pulses were initiated by the timer unit; their length and relative position on the time axis could be adjusted to any value. Usually the light pulse was initiated $100 \mathrm{~ms}$ before the start of the electric field pulse and was terminated, when the dichroism decay was complete.

Furthermore, the shape of the corrected transient $\left(\Delta A_{0}-\Delta A_{55}\right)$ closely corresponds to that of $\Delta A_{0^{-}}$ transients measured at low concentrations.

The magic angle effect observed at high concentrations seems to reflect field-induced interactions of purple membranes. This is suggested by the analogy of the $\Delta I_{55}$-transient shown in Figure 3 with those reported previously for lipid vesicles ${ }^{23}$ and DNA double helices. ${ }^{24}$ An analysis of the field-induced stationary amplitudes $\Delta I_{55}$ measured at different concentrations $\mathcal{C}_{\mathrm{BR}}$ indicates that there are two different contributions to the magic angle effect. The ratio $\Delta I_{55} / c_{\mathrm{BR}}$ increases with $c_{\mathrm{BR}}$, confirming the existence of an association process. If $\Delta I_{55}$ would be based on an intermolecular process exclusively, $\Delta I_{55} / c_{\mathrm{BR}}$ should approach zero at low concentrations. However, $\Delta I_{55}$ remains at a level of clearly detectable magnitude $\left(\Delta I_{55} / I_{55}^{0} \approx 10^{-3}\right.$ both

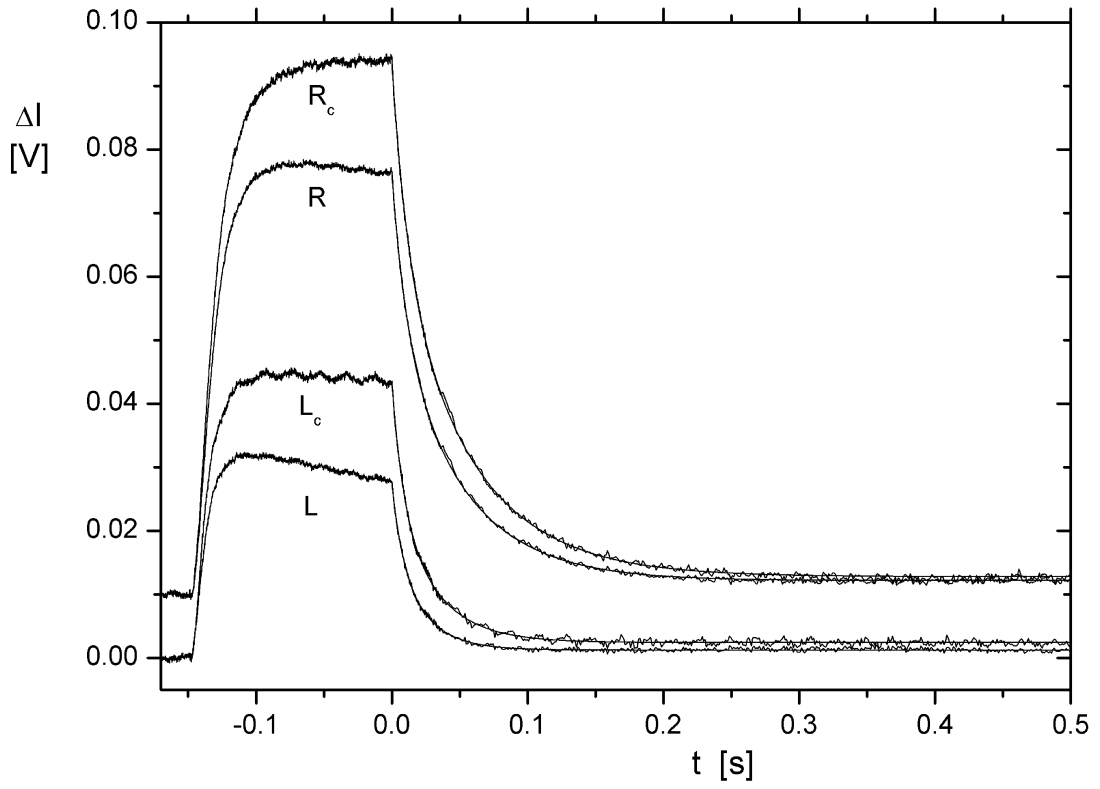

Figure 2. Change in transmission at $458 \mathrm{~nm} \Delta I$ by a suspension of D96N purple membranes due to an electric field pulse of $3.67 \mathrm{kV} \mathrm{m}^{-1}$ DC, measured with light polarized parallel to the field vector, in the resting state $(R)$ and the light-activated state $(\mathrm{L})$. The degree of lightactivation was $61 \%$. The time base is set to zero at the end of the electric field pulse. The fits of the decay curves by two exponentials (R: $\tau_{1}=10.6 \mathrm{~ms}, \tau_{2}=49.0 \mathrm{~ms}, A_{2}=$ $66.3 \%$; L: $\tau_{1}^{*}=7.2 \mathrm{~ms}, \tau_{2}^{*}=21.9 \mathrm{~ms}$, $\left.A_{2}^{*}=64.4 \%\right)$ can hardly be distinguished from the experimental curve. Transients obtained after correction by the magic angle effect are indicated by $\mathrm{R}_{\mathrm{c}}\left(\tau_{1}=11.6 \mathrm{~ms}\right.$, $\left.\tau_{2}=55.0 \mathrm{~ms}, A_{2}=66.0 \%\right)$ and $\mathrm{L}_{\mathrm{c}}$ $\left(\tau_{1}^{*}=8.03 \mathrm{~ms}, \quad \tau_{2}^{*}=29.3 \mathrm{~ms}\right.$, $\left.A_{2}^{*}=59.4 \%\right)$. The transients for the resting state $R$ and $R_{c}$ are shifted on the $\Delta I$-scale by $0.01 \mathrm{~V}$. Buffer A, $20{ }^{\circ} \mathrm{C}$, D96N concentration $2.49 \mu \mathrm{M}$. 


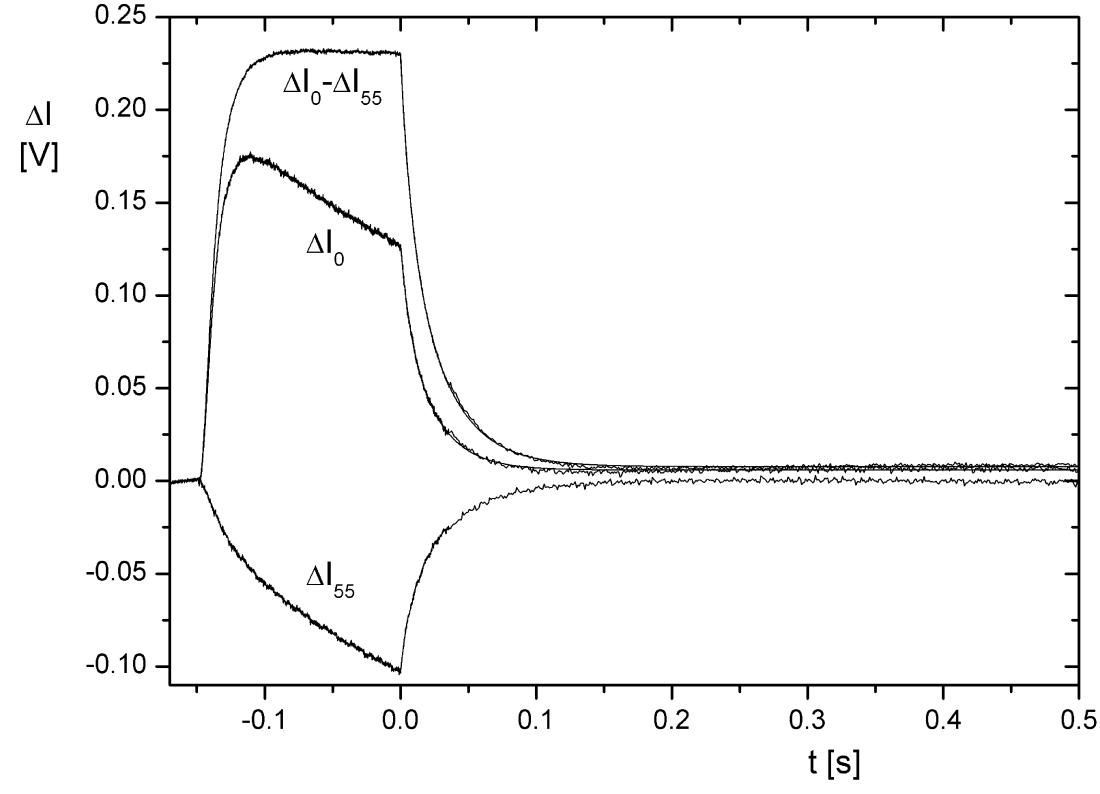

Figure 3. Change in transmission at $458 \mathrm{~nm}$ of polarized light $\Delta I_{\vartheta}$ by a suspension of $\mathrm{D} 96 \mathrm{~N}$ purple membranes due to an electric field pulse of $3.67 \mathrm{kV} \mathrm{m}^{-1}$ DC at different orientations of the polarization plane with respect to the field vector: $\Delta I_{0}$ parallel; $\Delta I_{55}$ magic angle. The transient denoted by $\Delta I_{0}-\Delta I_{55}$ is the difference between the transients at parallel and magic angle orientation. The degree of light-activation was $61 \%$. The time base is set to zero at the end of the electric field pulse. Least squares fits of the dichroism decays $\Delta I_{0}\left(\tau_{1}^{*}=6.0 \mathrm{~ms}\right.$, $\left.\tau_{2}^{*}=22.9 \mathrm{~ms}, A_{2}^{*}=75.6 \%\right)$ and $\Delta I_{0}-$ $\Delta I_{55} \quad\left(\tau_{1}^{*}=7.2 \mathrm{~ms}, \quad \tau_{2}^{*}=28.2 \mathrm{~ms}\right.$, $A_{2}^{*}=69.7 \%$ ) are represented by continuous lines. Buffer A, $20^{\circ} \mathrm{C}$, bacteriorhodopsin concentration $13.6 \mu \mathrm{M}$. for the resting and the light-activated state) in the range of low concentrations $c_{\mathrm{BR}}<3 \mu \mathrm{M}$. Furthermore, the special form of the $\Delta I_{55}$-transients illustrated in Figure 3 is not observed at low concentrations. Finally, the existence of a magic angle contribution in the low concentration range is indicated again by the ratio $\Delta I_{0} / \Delta I_{90}$, which is not consistent with the expected value -2 , when the $\Delta I$-values are used without correction, but approach the expected value -2 after correction with the magic angle effect (within experimental accuracy $\pm 20 \%$ ). All these observations indicate the existence of an intramolecular process contributing to the magic angle effect. The shape of the magic angle transients found at low concentrations is similar to that of the dichroism transients. Thus, the intra-molecular magic angle effect appears to be closely coupled to the process of orientation of the disks.

The magic angle effects are briefly described here without further discussion, because these effects were used to correct the dichroism transients discussed below, although the photo-induced changes are large and clearly visible without any correction (cf. Figure 2). The goal of this correction is optimal accuracy of the dichroism data over a broad range of concentrations.

The effect of the correction in the range of low concentrations is shown in Figure 2. After correction the risecurve for the light-activated state is converted from a special shape with a maximum in the transmission change to a standard shape with a continuous increase of transmission. In both cases the amplitude of the transmission change is increased. The correction also leads to some increase of the time constants, usually in the range of $10-20 \%$ (cf. legends to Figures 2 and 3 ). However, the strong acceleration effect induced by photo-activation is observed both in the raw and the corrected data.

\section{Rotational diffusion is accelerated during M-state}

The extremely large change of the dichroism decay indicates a major change in the global structure of the purple membranes. Apparently this change is due to formation of the M-state and, thus, the degree of transition to the M-state should be determined under the given experimental conditions. A simple procedure is based on determination of the light-induced absorbance change at $411 \mathrm{~nm}$, where the absorbance of the M-state is maximal. The change in the extinction coefficient at this wavelength due to formation of the M-state is $36 \times 10^{3} \mathrm{M}^{-1} \mathrm{~cm}^{-1}$, based on spectra given in the literature. ${ }^{25-27}$ Using these data, the degree of photo-activation has been determined for each experiment. In the examples presented in Figures 2 and 3, 61\% of the bacteriorhodopsin molecules were converted to the M-state. The ratio of the average time constants for the resting and the photo-activated state has been measured at different degrees of photo-activation. As shown in Figure 4 , the increase of this ratio with increasing photo-activation is more than linear. An exact interpretation of these data requires detailed hydrodynamic model calculations.

Most of the M-state is generated under the present experimental conditions within times of less than $100 \mathrm{~ms}$, as judged from the increase in the absorbance at $411 \mathrm{~nm}$. However, some change of the absorbance at $411 \mathrm{~nm}$ is observed up to the time range of about a second. Probably different M-sub-states are generated at different times of irradiation. However, measurements of the dichroism decay at different irradiation times between 0.1 second and 0.6 second did not reveal any difference within experimental accuracy of $\pm 10 \%$. If different sub-states are generated, these sub-states cannot be distinguished on the basis of the 


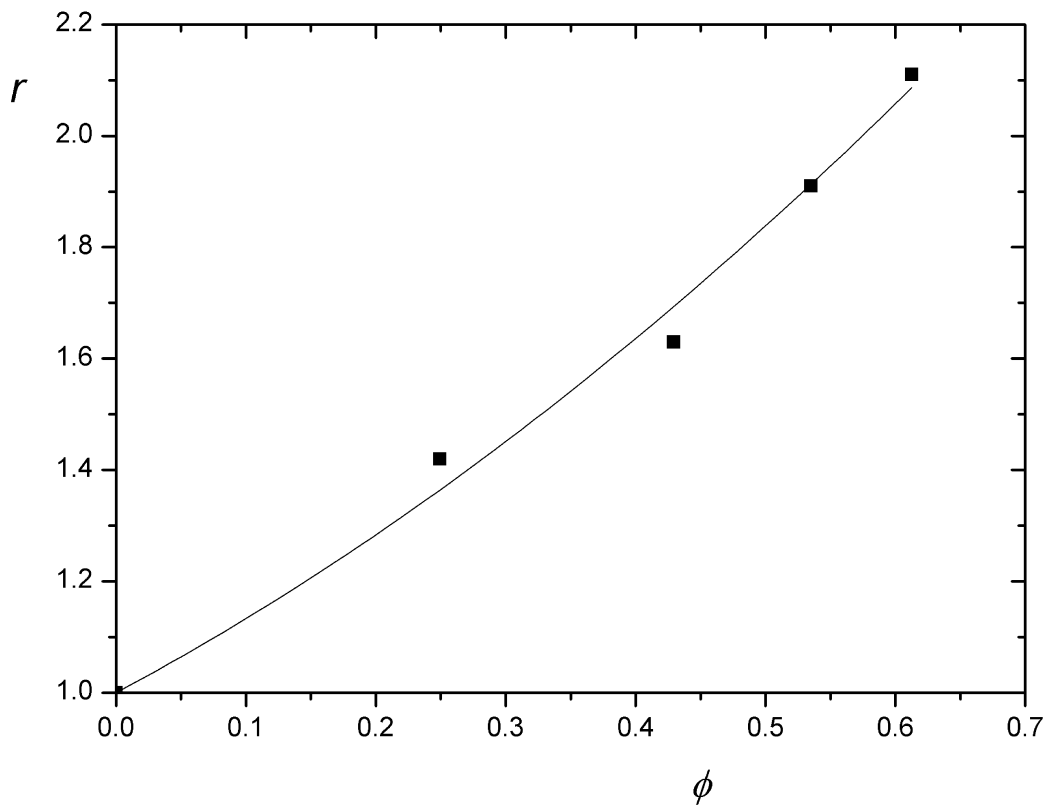

Figure 4. Ratio of the dichroism decay time constants $r$ obtained from $\tau_{2}$-values for the resting and the photo-activated state as a function of the degree of photo-activation $\phi$ (evaluation described in Results; buffer A, $20^{\circ} \mathrm{C}$, field pulses $3.67 \mathrm{kV} \mathrm{m}^{-1} \mathrm{DC}$, D96N concentration $3.44 \mu \mathrm{M})$. dichroism decay time. Based on the data collected for the photo-cycle, the late M-state should be accumulated under photo-stationary conditions.

Changes in dichroism decay time constants may result from different types of changes in the global structure. One of the possibilities is a change in the state of aggregation, which should be reflected by a dependence of the experimental time constants on the concentration of bacteriorhodopsin. Measurements in the range from $3 \mu \mathrm{M}$ to $14 \mu \mathrm{M}$ bacteriorhodopsin did not provide evidence for such concentration dependence, within experimental accuracy (cf. Figure 5). These data indicate that the light-induced change of the dichroism decay time constants is due to bending of the bacteriorhodopsin discs.

The lifetime of the state with the accelerated dichroism decay was analysed by measurements of this decay at different times $\Delta t$ after termination of light-activation. As shown in Figure 6, the integral dichroism decay time constant $\tau_{\mathrm{i}}$ increases in a time range very close to that observed for the decay of the M-state. The time constant obtained from the $\tau_{\mathrm{i}}=f(\Delta t)$ dependence is $500 \mathrm{~ms}$ and the time constant obtained under identical conditions from the absorbance at $411 \mathrm{~nm}$ for the decay of the $\mathrm{M}$-state is $750 \mathrm{~ms}$. The rather close correspondence shows that the state with the accelerated dichroism decay is equivalent with the M-state. An exact equivalence of the time constants is not expected, because the hydrodynamic changes are probably not linearly related to the amount of M-state.

The light-induced change of the dichroism decay was observed under various conditions. In buffer B

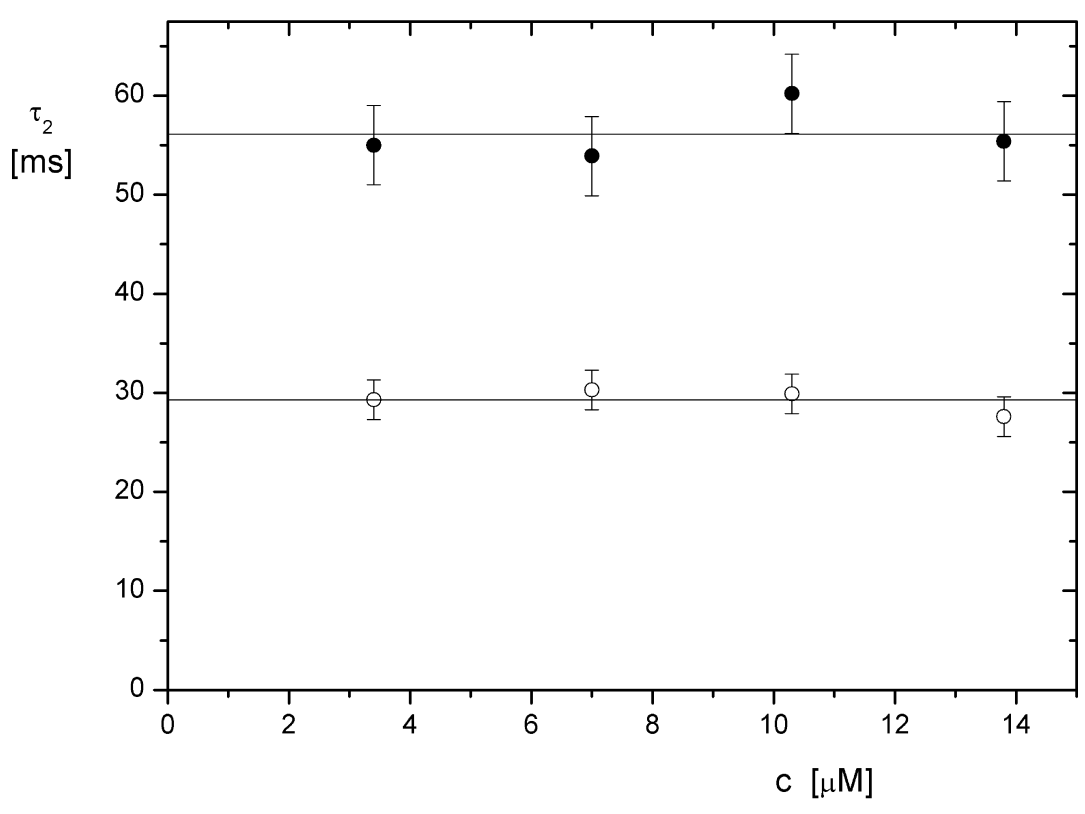

Figure 5. Dichroism decay time constant $\tau_{2}$ of D96N purple membranes (fractionated sample) measured at different bacteriorhodopsin concentrations in the resting state $(\bullet)$ and in the photo-activated state $(\mathrm{O})$. Buffer A, $20^{\circ} \mathrm{C}$, degree of photo-activation $61 \%$ (based on $\Delta I$ at $411 \mathrm{~nm}$; cf. Results), field pulses of $3.67 \mathrm{kV} \mathrm{m}^{-1} \mathrm{DC}$. 


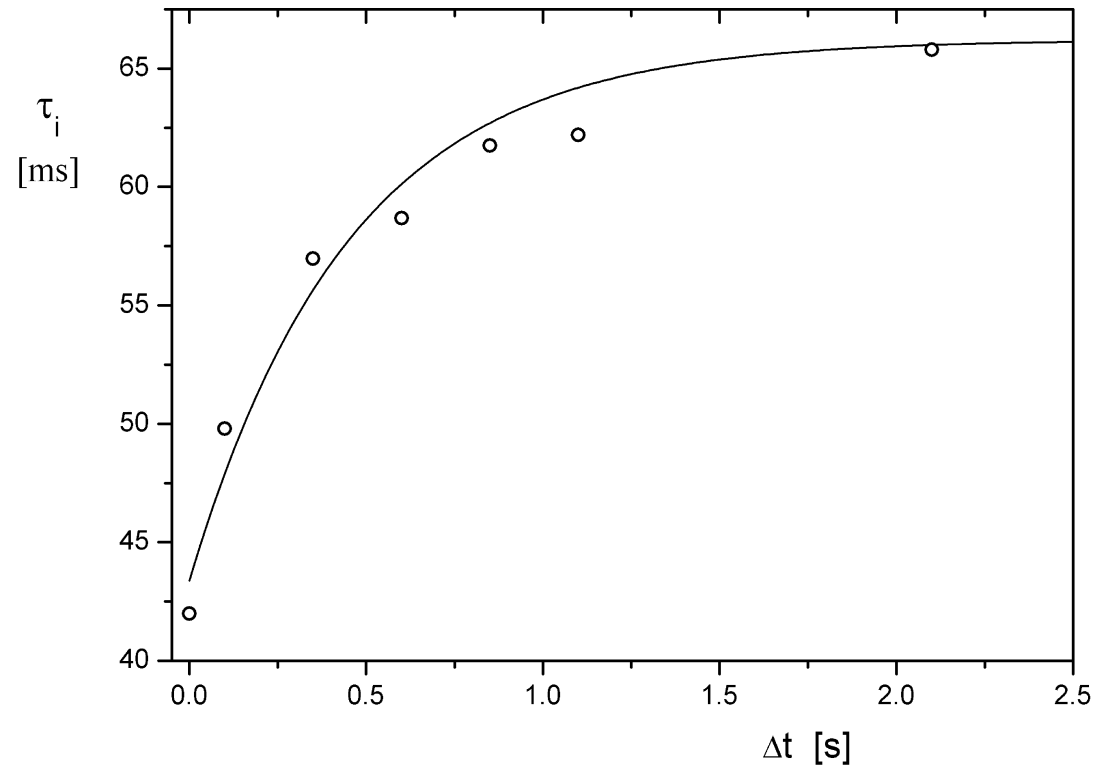

Figure 6. Integral decay time constant $\tau_{\mathrm{i}}$ for the light-activated state as a function of the time $\Delta t$ after termination of light-activation. The curved line represents a least squares exponential fit of the data with a time constant $\tau=500 \mathrm{~ms}$ (buffer $\mathrm{B}, \mathrm{pH}$ adjusted to 6.1 by addition of acetic acid, $20^{\circ} \mathrm{C}$, $6.14 \mu \mathrm{M} \quad \mathrm{D} 96 \mathrm{~N}, \quad 7.34 \mathrm{kV} \mathrm{m}^{-1} \quad \mathrm{DC}$ pulses). containing $\mathrm{Ca}^{2+}$ at $\mathrm{pH} 6.7$ the average decay time in the light-activated state $(\sim 70 \%$ M-state $)$ was smaller by a factor of 1.7 than that of the resting state. Reduction of $\mathrm{pH}$ to 5.1 by addition of acetic acid to this buffer lead to a decrease in this factor to 1.33 at $\sim 54 \% \mathrm{M}$-state. The $\mathrm{pH}$-dependence is partly due to a decrease in the lifetime of the Mstate with decreasing $\mathrm{pH}$. The available data suggest that light-induced bending is slightly less extensive in the presence of $\mathrm{Ca}^{2+}$ than in their absence.

Most of the dichroism decay time constants shown here were measured after pulses of $3.67 \mathrm{kV} \mathrm{m}^{-1}$. The results obtained from data after pulses of different field strengths are very similar, although some dependence on the field strength is observed, partly due to the fact that there was a distribution in the size of membrane fragments. The standard pulse of $3.67 \mathrm{kV} \mathrm{m}^{-1}$ represents a relatively low electric field strength, where the dichroism amplitude arrives already at a high level (cf. Figure 7). By this choice the accuracy of decay curves was sufficiently high and any perturbations by the electric field were reduced to a minimum.

\section{Stationary dichroism and photo-induced change of retinal angle}

As illustrated in Figure 2, photo-activation does not only change the dichroism decay but also the stationary dichroism amplitude. Measurements of these amplitudes at different field strengths (cf. Figure 7) show a decrease in the dichroism for the photo-activated state over a wide range of electric field strengths, whereas the overall shape of the field strength dependence is quite similar for the resting and the photo-activated state. These data were analysed by the orientation function for disks having a permanent dipole perpendicular to the plane and an induced dipole in the plane. ${ }^{28}$
The orientation of purple membranes ${ }^{9-19}$ in low DC-fields is determined by their permanent dipole moment, which is directed perpendicular to the membrane plane. The direction of orientation changes at high DC field strengths, ${ }^{19,28}$ where the induced dipole moment is dominant, causing preferential orientation along the membrane surface. AC-fields $(\geq 1 \mathrm{kHz})$ induce orientation by the induced dipole at any field strength, because the permanent dipole cannot follow rapid changes in the field direction. The induced dipole can change its direction very quickly, because it is based on changes in the ion distribution in the membrane plane, which are sufficiently fast to follow high frequencies up to the range of $\sim 1 \mathrm{MHz}$. The change in the mode of orientation from the permanent to the induced dipole is reflected in the sign of the dichroism.

The permanent and the induced dipole moments obtained by the quantitative analysis of the stationary DC-dichroism remain almost constant upon transition to the photo-activated state, whereas the limit dichroism is reduced by a factor of $\sim 1.9$ in the photo-activated state (Figure 7). However, the extrapolation to the limit of orientation may not be reliable enough, because the experimental dichroism values obtained by DC-field pulses are far away from the limit value. The experimental dichroism values induced by high AC-field pulses are much closer to the limit of complete orientation (cf. Figure 8) and, thus, extrapolation is more reliable. In this case, the limiting dichroism values are much larger and the difference between the resting and the photo-activated state is relatively small. These data indicate that the limit values of the dichroism depend on the mode of orientation and suggest that the mode of orientation affects the shape of particles (cf. Discussion).

The limit values of the dichroism are also dependent on the ion composition. In the presence of EDTA, used for chelating bivalent ions, the electric 


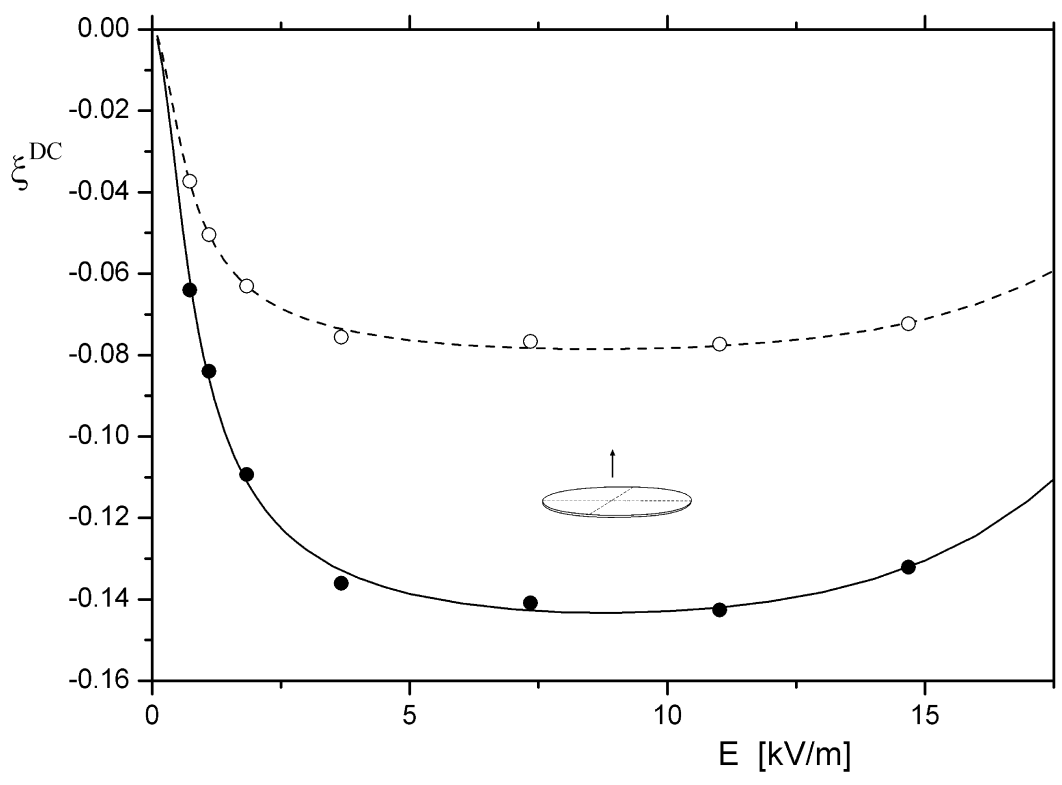

Figure 7. Stationary dichroism $\xi^{\mathrm{DC}}$ at $458 \mathrm{~nm}$ of $\mathrm{D} 96 \mathrm{~N}$ bacteriorhodopsin induced by DC-field pulses in the resting state $(\bullet)$ and in the photo-activated state $(O)$. The lines show least-squares fits to the data by the orientation function for disks having a permanent dipole perpendicular to the plane and an induced dipole in the plane. The fitted parameters for the resting and the photo-activated state are given without index and with index $*$, respectively: permanent dipole moment $\mu=1.92 \times 10^{-23} \mathrm{C} \mathrm{m} ; \quad \mu^{*}=2.18 \times$ $10^{-23} \mathrm{C} \mathrm{m}$; polarizability $\alpha=1.19 \times$ $10^{-27} \mathrm{C} \mathrm{m}^{2} \mathrm{~V}^{-1} ; \quad \alpha^{*}=1.37 \times$ $10^{-27} \mathrm{C} \mathrm{m}^{2} \mathrm{~V}^{-1}$; reduced limit dichroism $\quad \xi_{\infty}^{\mathrm{DC}}=0.083 ; \quad \xi_{\infty}^{\mathrm{DC} *}=$ 0.0448 ; buffer $\mathrm{B}, 20^{\circ} \mathrm{C}$; degree of photo-activation $69 \%$ (based on $\Delta I$ at $411 \mathrm{~nm}$; cf. Results), $6.06 \mu \mathrm{M}$ D96N. The inserted disk-symbol indicates the direction of preferential orientation with respect to the field vector represented by the arrow (cf. Results: Stationary dichroism and photo-induced change of the retinal angle).

dichroism is much higher, both in AC and DCmeasurements. The limit dichroism found in buffer A from AC-measurements is 0.424 for the resting state and 0.361 for the light-activated state $(61 \%$ $\mathrm{M})$. The corresponding values from DC-data are: 0.182 for the resting state and 0.112 for the lightactivated state. Compared to the rather large change in the limit dichroism, changes in the permanent dipole moment induced by removal of $\mathrm{Ca}^{2+}$ are negligible. Parameters obtained from fitting of stationary dichroism data to orientation functions are compiled in Table 1.

\section{Global structure in M-state of wt-bacteriorhodopsin is analogous to that of D96N}

The M-state of $\mathrm{D} 96 \mathrm{~N}$ is known for its particularly long lifetime and, thus, was selected for a convenient analysis. Because the effects observed for D96N may be partly due to the mutation, it is of interest to analyse the M-state of wt-bacteriorhodopsin. For a direct comparison, the electric dichroism of wt-bacteriorhodopsin was measured first under the same conditions as D96N. These

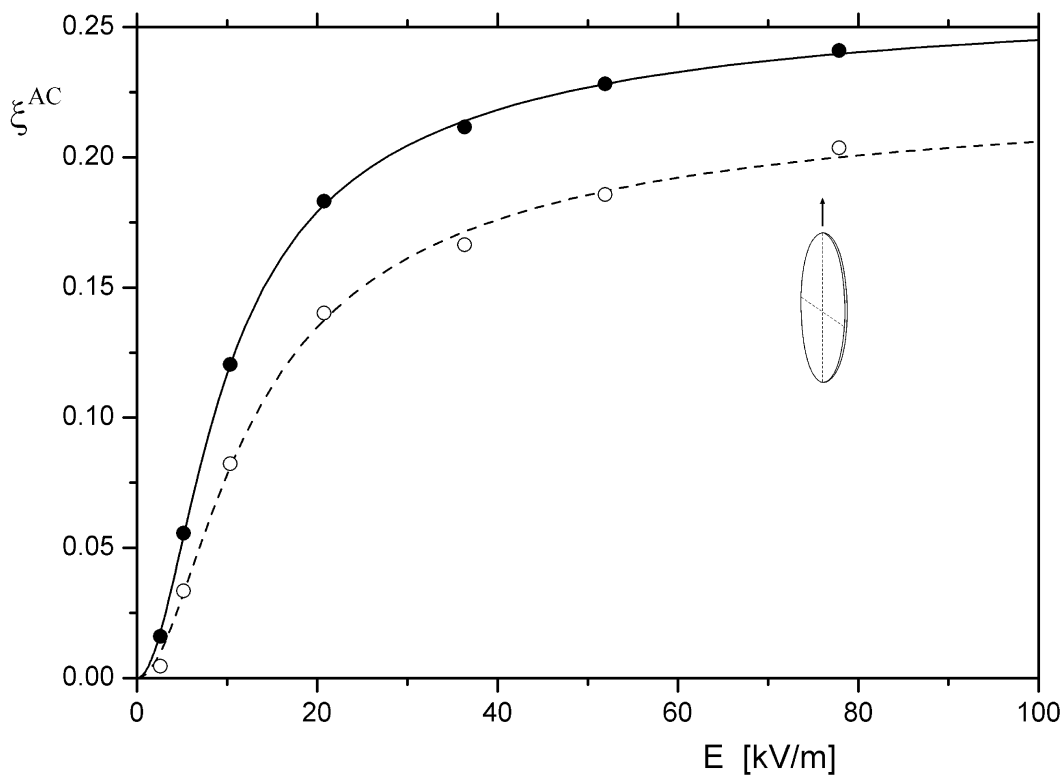

Figure 8. Stationary dichroism $\xi^{\mathrm{AC}}$ at $458 \mathrm{~nm}$ of $\mathrm{D} 96 \mathrm{~N}$ bacteriorhodopsin induced by AC-field pulses in the resting state $(\bullet)$ and in the photo-activated state $(O)$. The lines show least-squares fits to the data by the orientation function for disks having a permanent dipole perpendicular to the plane and a saturating induced dipole in the plane (unpublished results). The fitted parameters for the resting and the photo-activated state are given without index and with index $*$, respectively: polarizability $\alpha=3.31 \times 10^{-28} \mathrm{C} \mathrm{m}^{2} \mathrm{~V}^{-1} ; \alpha^{*}=$ $2.10 \times 10^{-28} \mathrm{C} \mathrm{m}^{2} \mathrm{~V}^{-1}$; limit field strength $\quad E_{0}=4.79 \mathrm{kV} \mathrm{m}^{-1} ; \quad E_{0}^{*}=$ $5.55 \mathrm{kV} \mathrm{m}^{-1}$; reduced limit dichroism $\xi_{\infty}^{\mathrm{AC}}=0.265 ; \xi_{\infty}^{\mathrm{AC} *}=0.229 ;$ buffer $\mathrm{B}, 20{ }^{\circ} \mathrm{C}$; degree of photo-activation $71 \%$ (based on $\Delta I$ at $411 \mathrm{~nm}$; cf. Results), $6.13 \mu \mathrm{M}$ D96N. The inserted disk-symbol indicates the direction of preferential orientation with respect to the field vector represented by the arrow (cf. Results: Stationary dichroism and photo-induced change of the retinal angle). 
Table 1. Parameters obtained for D96N purple membranes by fitting of stationary dichroism data to orientation functions

\begin{tabular}{|c|c|c|c|c|c|c|}
\hline Buffer & $E$-field & $\% \mathrm{M}$ & $\mu\left(10^{-24} \mathrm{C} \mathrm{m}\right)$ & $\alpha\left(10^{-28} \mathrm{C} \mathrm{m}^{2} \mathrm{~V}^{-1}\right)$ & $E_{0}\left(\mathrm{kV} \mathrm{m}^{-1}\right)$ & $\xi_{\infty}$ \\
\hline B & DC & 0 & 19.2 & 11.9 & & 0.083 \\
\hline B & DC & 69 & 21.8 & 13.7 & & 0.0448 \\
\hline B & $\mathrm{AC}$ & 0 & & 3.31 & 4.79 & 0.265 \\
\hline B & AC & 71 & & 2.1 & 5.55 & 0.229 \\
\hline A & DC & 0 & 16.5 & 5.72 & & 0.182 \\
\hline A & DC & 61 & 15.2 & 4.74 & & 0.112 \\
\hline A & $\mathrm{AC}$ & 0 & & 1.58 & 6.09 & 0.424 \\
\hline A & $A C$ & 61 & & 0.84 & 7.12 & 0.361 \\
\hline
\end{tabular}

DC-data were fitted to the orientation function described by Shah; ${ }^{28}$ AC-data were fitted to a modified Shah function with a saturating induced dipole moment (unpublished results). The resting state is denoted by $0 \% \mathrm{M}$ and the light-activated state is specified by the percentage of M-state (see the text). $\mu$, permanent dipole moment; $\alpha$, polarizability; $E_{0}$, saturation field strength; $\xi_{\infty}$, limit dichroism; $20^{\circ} \mathrm{C}$, estimated accuracy $\pm 10 \%$.

measurements at $\mathrm{pH}$-values around 7 did not reveal large changes in the dichroism decay or of any other dichroism parameter during photo-activation. Because the lifetime of the M-state is extended at alkaline $\mathrm{pH}$, wt-bacteriorhodopsin was then analysed at $\mathrm{pH}$-values of $7.8,8.3$ and 9.3 , where a sufficiently high degree of molecules ( $\sim 18.2 \%, 23.6 \%$ and $32.3 \%$, respectively) was driven to the $\mathrm{M}$-state by photo-activation. Under these conditions the dichroism decay was accelerated in the light-activated state by factors of 1.11, 1.19 and 1.28 , respectively. These data demonstrate a continuous increase in the acceleration effect with increasing $\mathrm{pH}$. The magnitude of the effect is similar to that observed for D96N at the same degree of photo-activation (cf. Figure 4). The dichroism decay of the wt-bacteriorhodopsin sample could be described by single exponentials at a satisfactory accuracy. The average decay time constant in the resting state was $52.4 \mathrm{~ms}$.

\section{Bead model simulations}

Dichroism decay time constants are known to be very sensitive indicators of hydrodynamic dimensions. The rotation time constant $\tau_{R}$ of circular disks $^{29}$ increases with the cube of their diameter $d$ :

$$
\tau_{\mathrm{R}}=\frac{2 \eta d^{3}}{9 k T}
$$

where $\eta$ is the viscosity and $k T$ the thermal energy. Thus, for a disk with $d=1 \mu \mathrm{m}$ we get $\tau_{\mathrm{R}}=55 \mathrm{~ms}$ and with $d=0.5 \mu \mathrm{m} \quad \tau_{\mathrm{R}}=6.9 \mathrm{~ms}$ (in water at $20{ }^{\circ} \mathrm{C}$ ). However, there is no simple analytical relation to deduce the bending angle of a circular disk from a reduced time constant of rotational diffusion. Bead model simulations ${ }^{30,31}$ are required to get this type of information. The global structure of a disk was modelled by an assembly a spherical beads, as shown in Figure 9. The diameter of the flat circular disk illustrated in Figure 9(a) is $990 \mathrm{~nm}$. This disk was bent in a cylindrical mode with different bending radii. The orientation mode was defined by the permanent dipole moment, which is in perpendicular direction to the disk plane. If there is a net charge distributed on the disk surface, bending will contribute to the permanent dipole in this direction. The simulated dichroism decay curves can be represented by single exponentials at a sufficient accuracy, although there are contributions from five exponentials. The
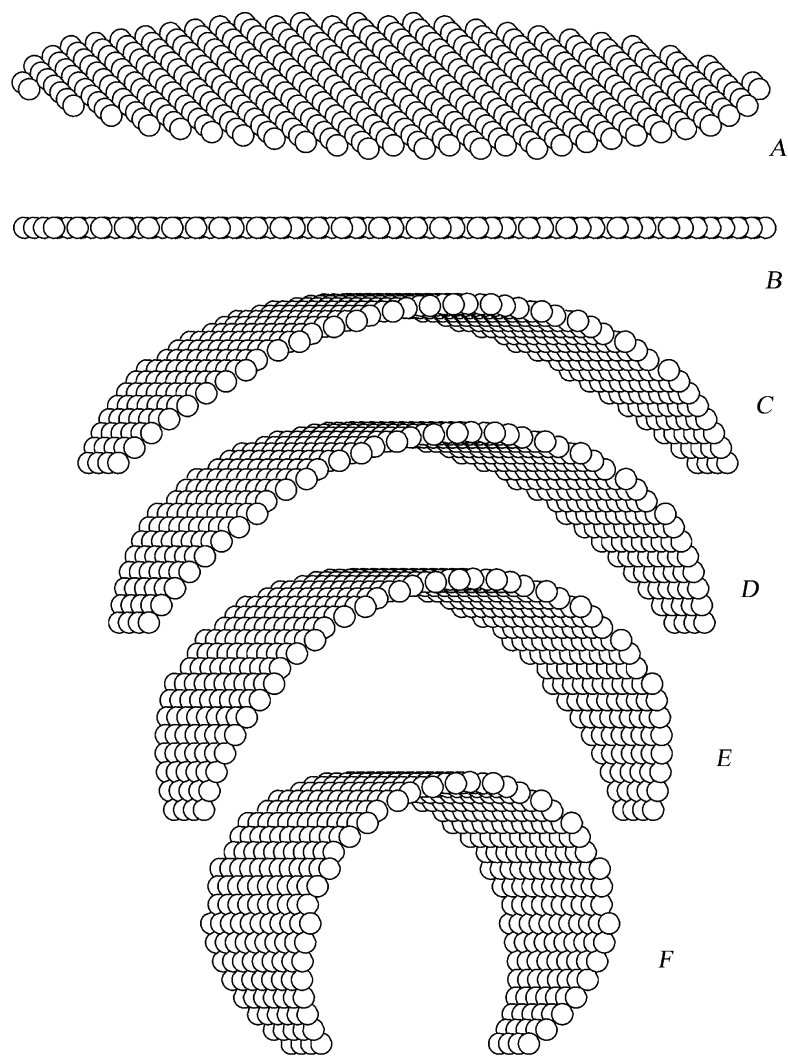

Figure 9. Bead models of a circular disk with a diameter of $990 \mathrm{~nm}$ at different bending angles. The model is constructed out of 399 beads with a radius of $14 \mathrm{~nm}$. The views (a) and (b) show the planar disk in two different orientations. The views (c)-(f) show different forms, which are bent to the surface of cylinders with radii of $500 \mathrm{~nm}, 400 \mathrm{~nm}, 300 \mathrm{~nm}$, and $200 \mathrm{~nm}$. The views (b)-(f) are generated using the same rotational angles. The simulated dichroism decay time constants are 48.9, 39.6, $35.5,28.7$ and $17.6 \mathrm{~ms}$ for bending radii of $\infty, 500,400$, 300 and $200 \mathrm{~nm}$, respectively. 
fitted single exponential time constants are 48.9, $39.6,35.5,28.7$ and $17.6 \mathrm{~ms}$ for bending radii of $\infty$, 500, 400, 300 and $200 \mathrm{~nm}$, respectively. A comparison of experimental and simulated time constants demonstrates that the degree of curvature induced by light-activation is very large. The bending radius at high degrees of photo-activation in the range of $61 \%$ is in the order of magnitude of $250 \mathrm{~nm}$

\section{Discussion}

Molecular electro-optical techniques are useful to detect both global and local changes in macromolecular structures in solution at a particularly high sensitivity. The information on structure can be obtained within a time determined by its rotational diffusion time constant. Thus, structures of intermediates can be characterized by molecular electro-optics in solution at a relatively high time resolution. The information is provided on: (1) the charge distribution indicated by the dipole moment, (2) the arrangement of chromophores indicated by the limit dichroism and (3) the size and shape of the global structure indicated by the time constant(s) of rotational diffusion.

\section{Charge distribution and dipole moment}

In the case of bacteriorhodopsin the information on the charge distribution is of particular interest, because proton motion during photo-activation may change this distribution. A simple model calculation indicates the order of magnitude: motion of a proton from the intra- to the extra-cellular side may change the dipole moment by $1 / 2 \times e \times$ $50 \times 10^{-10} \mathrm{C} \mathrm{m}=120 \mathrm{D}$ per protein molecule. This change is considerable with respect to the experimental dipole moment of $\sim 50 \mathrm{D}$ per monomer, but relatively small compared to the dipole moment calculated from the crystal structure of the protein in the order of $600-800$ D. ${ }^{19,32}$ The effect of proton motion on the dipole moment may also be counterbalanced by motion of charged residues of the protein. A recent investigation indicates that the overall dipole moment of the protein is compensated to a large extent, apparently by a nonsymmetric distribution of charges on lipid residues. ${ }^{32}$ The overall permanent dipole moments obtained in the present investigation suggest that the change in the charge distribution between the resting and the M-state is negligible. However, a dipole change resulting from displacement of some residue(s) may be compensated by rearrangement of some other residue(s). Furthermore, the considerable change of shape during transition to the M-state indicated by the dichroism decay prevents a detailed comparison of molecular dipole components, because bending of charged objects may provide a large contribution to the overall dipole moment. ${ }^{33}$

\section{Retinal angle and limit dichroism}

The orientation of purple membranes is driven by AC-fields rather close to the limit of perfect orientation, whereas the orientation is far from this limit in the case of standard DC-fields. Thus, the limit dichroism can be obtained at a much higher reliability from AC-data. The example given in Figure 8 shows that the limit dichroism of the photo-activated state is smaller than that of the resting state by $\sim 15 \%$, corresponding to a relatively small change of the retinal orientation. A completely different result is obtained from DCdata, indicating a large reduction of the dichroism in the photo-activated state by a factor of 2 .

The difference between the limit dichroism $\xi_{\infty}^{\mathrm{DC}}$ obtained from DC and $\xi_{\infty}^{\mathrm{AC}}$ from AC-measurements is mainly due to the fact that the available orientation function does not represent the various effects contributing to the dichroism accurately enough. First of all, usual bacteriorhodopsin preparations include different particle sizes and shapes. Furthermore, the shape of the particles is not constant but subject to bending. The degree of bending is probably affected by various factors, including the electric field. Model calculations demonstrated that bending of objects with a high charge density leads to considerable dipole moments. ${ }^{33}$ Fieldinduced bending of purple membranes must be expected as well as field-induced stretching. The processes of bending and stretching are dependent on the details of the experimental conditions. When the disks are oriented mainly by their induced dipole moment, stretching is expected, because the induced dipole is maximal in the stretched state. Thus, stretching must be expected under AC-pulses in general and under DC-pulses of high field strengths. The energy term due to the permanent dipole moment is dominant at low field strengths of DC-pulses. In this domain, the dipole moment may be increased by bending and, thus, bending may be enhanced. Because the experimental dipole moments of the resting and of the M-state are close to each other, the degree of field-induced bending or stretching should be similar.

Due to the different effects contributing to the stationary dichroism, a complete quantitative interpretation cannot be presented yet. Apparently high AC-fields induce stretching of purple membranes and, thus, the limit dichroism obtained from AC-measurements for the resting and the Mstate are relatively close to each other. The difference in the $\xi_{\infty}^{\mathrm{AC}}$-values between resting and lightactivated state is probably mainly due to a difference in the local orientation of retinal in the protein. At low DC-field strengths field-induced bending is expected to be negligible or at least similar for the resting and the light-activated state, because the permanent dipole moments are of similar magnitude. Thus, the large difference in the stationary dichroism between the resting and the light-activated state observed at low DC-fields 
(Figure 7) is mainly attributed to light-induced bending of membrane fragments.

Based on this assignment of factors contributing to the dichroism amplitude, we may proceed in the analysis with the assumption that the disks are stretched out at high AC-field strengths. Assuming a simple flat structure of the discs, the angle of the transition dipole moment derived from $\xi_{\infty}^{\mathrm{AC}}$ is $62.3^{\circ}$ for the resting state and $61.2^{\circ}$ for the photo-activated state $(71 \% \mathrm{M})$ in the presence of $\mathrm{Ca}^{2+}$. The higher values of the limit dichroism found in the presence of EDTA provide retinal angles of $67.6^{\circ}$ for the resting state and $65.4^{\circ}$ for the photo-activated state $(61 \% \mathrm{M})$.

The orientation of retinal has been analysed by many different methods (for a summary cf. Ref. 34). The present data obtained from AC-measurements in the presence of EDTA are in the same order of magnitude as results reported in the literature. The polyene chain direction in the crystal structure is $69.8^{\circ}$ with respect to the membrane normal. ${ }^{35}$ This angle is decreased in the M-state by $2.2^{\circ}$ according to Schertler et al. ${ }^{36}$ whereas Luecke et $a{ }^{35}$ report a reduction by $9.2^{\circ}$. The difference may be due to the analysis of different crystal forms in these investigations. Linear extrapolation of the dichroism change found in the present measurements to $100 \% \mathrm{M}$-state provides a change of the retinal angle of $3.6^{\circ}$ (buffer A containing EDTA). The dependence of the retinal angle on the ion composition found in the present investigation has not been reported previously. This dependence suggests the existence of some ionbinding site(s) close to the retinal group. However, high resolution structures of bacteriorhodopsin did not provide evidence for such binding site(s). Thus, the dependence of the retinal angle on the ion composition may be based on ion binding to surface residues, which induces a change in the $\mathrm{pK}$ of internal carboxyl groups.

\section{Decay time constants indicate strong light-induced bending}

The most remarkable effect detected in the present investigation is the large decrease in the dichroism decay time constants in the photo-activated state. This decrease clearly demonstrates a major change in the global structure. One of the potential explanations is a photo-induced dissociation reaction of the disks. However, dissociation should be reflected by some concentration dependence of the time constants that was not detected in the experimental data. Another argument against photo-dissociation is provided by the parameters of the bi-exponential fits. If there would be photo-dissociation, the amplitude of the faster process should increase upon photo-activation, because the fraction of smaller particles should increase. Due to the extreme dependence of dichroism decay times on particle sizes (cf. equation (1)), this effect associated with dissociation should be easily detectable, but has not been observed. Thus, the available experimental data support an interpretation by photo-induced bending.

The extent of bending induced by photo-activation is considerable as judged by the large change in the dichroism decay time. However, a quantitative assignment of bending angles requires hydrodynamic model calculations. One of the difficulties for an exact assignment is the heterogeneity of the sample preparation with respect to size and shape. However, an approximate assignment may be based on disk models with a circular shape. Using equation (1) the experimental rotational time constants $\tau_{1}=11.6 \mathrm{~ms}, \tau_{2}=55.0 \mathrm{~ms}$ for the resting state (Figure 2) provide average disk sizes of $0.6 \mu \mathrm{m}$ and $1 \mu \mathrm{m}$ as major components of the distribution. These numbers illustrate the fact that relatively small changes in disk dimensions result in large changes in rotation time constants.

The change of $\tau_{2}$ upon photo-activation is much larger than that of $\tau_{1}$. This result is consistent with bending. Both small and large particles are driven to the same degree of photo-activation and, thus, their bending radius should be equivalent. Under these conditions the effect of bending on the hydrodynamic dimensions increases strongly with the size of the particles. The bead model simulations on a disk with $\sim 1 \mu \mathrm{m}$ diameter clearly demonstrate that the degree of photo-induced bending is extensive. The acceleration of rotational diffusion by a factor $\sim 2$ observed for particles of this size at $61 \%$ photo-activation corresponds to a bending radius of $\sim 250 \mathrm{~nm}$.

Because photo-activation cannot be driven to completion, it may be suspected that different time constants of the dichroism decay may reflect activated and non-activated molecules. However, each membrane fragment contains a high number of monomers (on average $\sim 50,000$ ) and each of these is activated with the same probability. Thus, the average degree of photo-activation for the whole fragment population should be valid for each individual membrane fragment. Selective photo-activation of individual membrane fragments would require a high degree of cooperativity, which has not been found for bacteriorhodopsin.

Among the potential contributions to lightinduced bending of purple membranes may be accumulation of protons on the extracellular side of the membrane. An argument against this interpretation comes from the decay of bending after termination of light-activation. As shown in Figure 6, the experimental time constant for the decay of bending $(500 \mathrm{~ms})$ is more than two orders of magnitude larger than that for transfer of protons to the bulk solution. ${ }^{37-40}$ Thus, asymmetry of $\mathrm{pH}$ is not the basis of the bending effect. The rather close correspondence of time constants for the decays of bending and $\mathrm{M}$-state indicates that the structure of the M-state is the source of bending. Bending is suppressed due to packing in the crystalline state of the samples, but is without these limits in solution. 


\section{Comparison with literature data}

"Transient changes in the curvature of the purple membrane" have been discussed by Czege \& Rheinisch ${ }^{20,21}$ based on measurements of light-scattering intensities in the $\mathrm{pH}$-range $4-11$. These authors used wt-bacteriorhodopsin and short light pulses for activation, whereas a photo-stationary state was analysed in the present investigation. Czege \& Rheinisch found only relatively small changes in scattering up to $3 \%$. Their changes are maximal at $\mathrm{pH} 8$ and decrease at $\mathrm{pH}>8$. Thus, the $\mathrm{pH}$-dependence described by Czege \& Rheinisch is very different from that found in the present investigation and is not consistent with an assignment of bending to the M-state. Furthermore Czege \& Rheinisch ${ }^{20}$ report a decreased degree of bending upon photo-activation for $\mathrm{pH}>5$. Thus, not only the magnitude of the effect and its $\mathrm{pH}-$ dependence but also the direction of the effect is different. These differences are probably mainly due to the fact that light-scattering intensities are not nearly as sensitive with respect to changes in shape as rotational diffusion. Furthermore, Czege \& Rheinisch did not use the D96N-mutant, where the effect can be observed more easily than with wt-bacteriorhodopsin. Czege \& Rheinisch did not report their degree of photo-activation, which would be essential for any comparison, because the effect of bending is expected to be non-linear. Finally, as mentioned also by Czege \& Rheinisch, there are problems in the interpretation of the light-scattering intensities.

\section{Biological function}

Purple membranes are usually considered in the literature to be planar disk structures, although the general shape of Halobacteria implies a high degree of bending. According to Stoeckenius et al. ${ }^{41}$ the cells of Halobacterium salinarium "usually are rods of approximately $0.5 \mu \mathrm{m}$ diameter and 4-10 $\mu \mathrm{m}$ length". Thus, purple membrane patches in vivo are strongly bent to cylindrical forms. The curvature found in the present experiments at $60 \%$ photo-activation happens to be close to the natural one in the membrane of Halobacteria. Apparently the curvature can be modulated by various factors. The surface charge density is clearly one of the essential factors. Other factors remain to be investigated as well as the energy required for bending. It is possible, for example, that purple membranes in their isolated state are released from "bending strain" imposed by the in vivo situation. The present results clearly demonstrate that photo-excitation changes the balance of factors contributing to bending to a large extent and, thus, lightinduces a bending signal. The molecular basis for bending of purple membranes in the photo-activated state is expected to be a change of the protein structure. Changes in bacteriorhodopsin structure during the photocycle were analysed and discussed by several authors. The findings obtained by different approaches are contradictory. Subramaniam \& Henderson ${ }^{42}$ using electron crystallography found particularly large movements within helix $F$ (up to 2-3A), whereas the X-ray analysis of crystals did not show this transition. . $^{35,43}$ Probably "the main structural change is inhibited or muted in the three-dimensional crystals". ${ }^{42,44} \mathrm{As}$ shown in Figure 10, the changes reported by Subramaniam \& Henderson are equivalent with a wedge-like deformation of the protein in the trans-membrane direction during the M-state. The effect of this deformation on the global structure of purple membranes has not been considered yet. It is obvious, however, that a wedge-like deformation of the monomer must be amplified to a high degree of bending in purple membranes.

A biological role of bending in the light-induced state cannot be specified yet. It is possible that bending of purple membranes is used as a "mechanical" signal in the membrane. Although signal

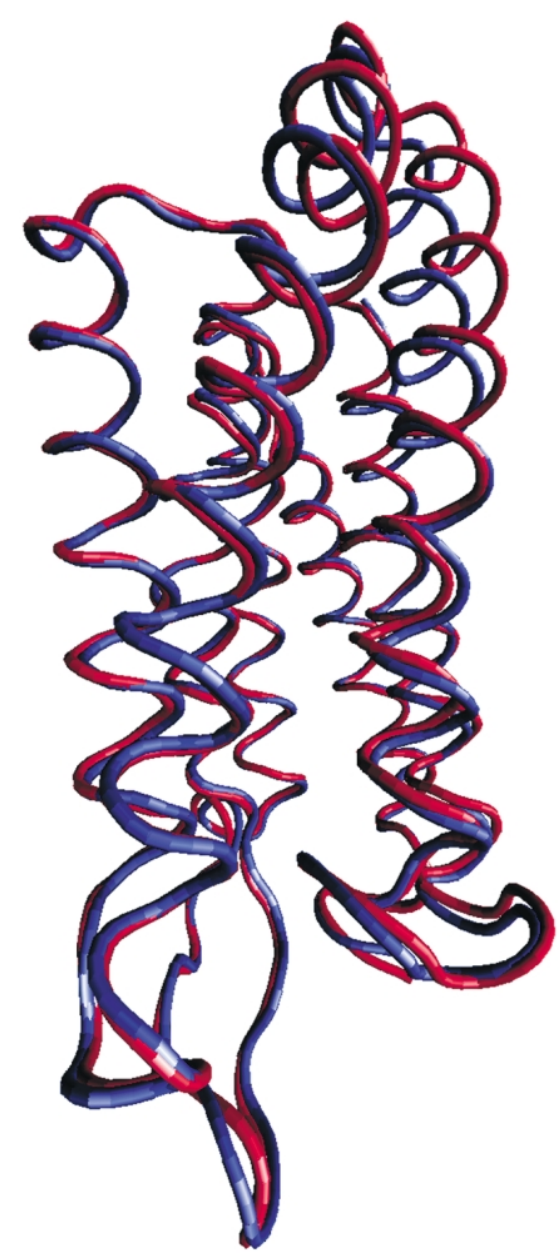

Figure 10. View of the $\alpha$-helical structure of bacteriorhodopsin in the resting state (blue, 1FBB) and in the "late" M-state (red, 1FBK) according to Subramaniam \& Henderson. ${ }^{42}$ Movements of the helices F and G open the proton channel at the cytoplasmic side and generate a wedge-like structure. The coordinates were loaded from the Protein Data Bank; the Figure was generated with VMD. ${ }^{48}$ 
action via bending remains speculative at the present state of knowledge, the unusually large lightinduced bending found in purple membranes suggests that it is worthwhile to study this possibility in more detail. The existence of mechanosensitive ion channels in bacteria ${ }^{45}$ demonstrates that signals of this type can be detected and converted.

\section{Materials and Methods}

Wild-type (wt) bacteriorhodopsin was prepared by standard procedures ${ }^{46}$ from $H$. salinarium, provided by Dr J. Tittor. The D96N sample was kindly donated by Dr J. Tittor. The membrane fragments were dialysed extensively against the buffers used for measurements. Buffer A: $1 \mathrm{mM} \mathrm{NaClO}, 1 \mathrm{mM}$ sodium cacodylate $(\mathrm{pH}$ 6.7), $0.2 \mathrm{mM}$ EDTA; buffer B: $1 \mathrm{mM} \mathrm{NaClO}_{4}, 1 \mathrm{mM}$ sodium cacodylate $\left(\mathrm{pH}\right.$ 6.7), $0.2 \mathrm{mM} \mathrm{Ca}\left(\mathrm{ClO}_{4}\right)_{2}$. The measurements with wt-bacteriorhodopsin at $\mathrm{pH}$-values $>7$ were performed in the buffers: $3 \mathrm{mM}$ Tris $-\mathrm{HCl}(\mathrm{pH}$ $7.82), 0.2 \mathrm{mM} \mathrm{Ca}\left(\mathrm{ClO}_{4}\right)_{2} ; 3 \mathrm{mM}$ Tris- $\mathrm{HCl}(\mathrm{pH} 8.27)$ $0.2 \mathrm{mM} \mathrm{Ca}\left(\mathrm{ClO}_{4}\right)_{2} ; 2 \mathrm{mM}$ sodium borate $(\mathrm{pH}$ 9.32), $0.2 \mathrm{mM} \mathrm{Ca}\left(\mathrm{ClO}_{4}\right)_{2}$.To suppress aggregation, the samples were sonicated in a Bransonic 220 sonicator for three minutes before electro-optical measurements. The samples were light-adapted by irradiation with a cold light source.

Combined application of electric field pulses and of intensive light pulses to a given solution requires a special instrument, which allows independent initiation of the pulses. An instrument of this type was constructed using the units of a standard field jump instrument ${ }^{8,19}$ together with a cell that has been designed for fluorescence detection. ${ }^{47}$ The cell with Pt-electrodes at the top and bottom is equipped with two standard conical quartz windows for absorbance measurements and two conical windows with a large aperture for fluorescence measurements. The light path for fluorescence measurements, which is arranged in perpendicular direction to the light path for absorbance measurements, is used in the present application for irradiation of light to generate the M-state of bacteriorhodopsin. Two $600 \mathrm{~W}$ lamps from Hanovia were used as light sources (one from the left and the other from the right side); the irradiation time was determined by electromagnetic shutters. The wavelength of the irradiation light was limited by cut-off filters to $\lambda>520 \mathrm{~nm}$. Polarization filters oriented at an angle of $55^{\circ}$ with respect to the electric field vector were used for isotropic excitation of bacteriorhodopsin. DC and AC-pulses were generated by an arbitrary waveform generator Tektronix AWG 5105 together with a Fluke amplifier 5205A. A scheme of the instrument is presented in Figure 1.

The dichroism decay curves of $\mathrm{D} 96 \mathrm{~N}$ bacteriorhodopsin required two exponentials for accurate fits. Because amplitudes and time constants of such fits may vary over a relatively wide range, unless the accuracy of the data is very high, in some cases integral time constants $\tau_{i}$ were used as defined by:

$$
\tau_{\mathrm{i}}=\frac{A_{1}}{A_{1}+A_{2}} \tau_{1}+\frac{A_{2}}{A_{1}+A_{2}} \tau_{2}
$$

where $A_{1}, A_{2}, \tau_{1}$ and $\tau_{2}$ are the amplitudes and the time constants of the first and the second exponential, respectively.

\section{Acknowledgements}

The technical assistance of Jürgen Wawrzinek is gratefully acknowledged.

\section{References}

1. Stoeckenius, W. \& Bogomolni, R. A. (1982). Bacteriorhodopsin and related pigments of halobacteria. Annu. Rev. Biochem. 52, 587-616.

2. Ottolenghi, M., Sheves, M., eds (1995). Isr. J. Chem. 35 193-515.

3. Haupts, U., Tittor, J. \& Oesterhelt, D. (1999). Closing in on bacteriorhodopsin: progress in understanding the molecule. Annu. Rev. Biophys. Biomol. Struct. 28, 367-399.

4. Lanyi, J. K., ed. (2000). Bacteriorhodopsin. Biochim. Biophys. Acta, 1460, 1-239.

5. Patzelt, H., Simon, B., terLaak, A., Kessler, B., Kühne, R., Schmieder, P. et al. (2002). The structures of the active center in dark-adapted bacteriorhodopsin by solution-state NMR spectroscopy. Proc. Natl Acad. Sci. USA, 99, 9765-9770.

6. Lanyi, J. K. \& Schobert, B. (2002). Crystallographic structure of the retinal and the protein after deprotonation of the Schiff base: the switch in the bacteriorhodopsin photocycle. J. Mol. Biol. 321, 727-737.

7. Fredericq, E. \& Houssier, C. (1973). Electric Dichroism and Electric Birefringence, Clarendon, Oxford.

8. Porschke, D. (2001). Molecular electro-optics. In Protein Ligand Interactions: Hydrodynamics and Calorimetry-A Practical Approach (Harding, S. E. \& Chowdhry, B. Z., eds), pp. 197-221, Oxford University Press, Oxford.

9. Keszthelyi, L. (1980). Orientation of membrane fragments by electric field. Biochim. Biophys. Acta, 598, 429-436.

10. Kimura, Y., Ikegami, A., Ohno, K., Saigo, S. \& Takeuchi, Y. (1981). Electric dichroism of purple membrane suspensions. Photochem. Photobiol. 33, 435-439.

11. Kimura, Y., Fujiwara, M. \& Ikegami, A. (1984). Anisotropic electric properties of purple membrane and their change during the photoreaction cycle. Biophys. J. 45, 615-625.

12. Barabas, K., Der, A., Dancshazy, Z., Ormos, P. Keszthelyi, L. \& Marden, M. (1983). Electro-optical measurements on aqueous suspension of purple membrane from halobacterium halobium. Biophys. J. 43, 5-11.

13. Kahn, L. D. \& Tu, S. I. (1984). Electric birefringence study of the purple membrane of Halobacterium halobium. Biopolymers, 23, 707-718.

14. Stoylov, S. P., Todorov, G. \& Zhivkov, A. (1984). Effect of external electric fields on membrane proteins: the bacteriorhodopsin. Bioelectrochem. Bioenerg. 12, 49-55.

15. Papp, E., Fricsovszky, G. \& Meszena, G. (1986). Electrodichroism of purple membrane: ionic strength dependence. Biophys. J. 49, 1089-1100.

16. Taneva, S. G., Jordanova, N. \& Petkanchin, I. B. (1992). Electro-optical investigation of lipid depleted purple membranes. Biophys. Chem. 44, 91-97.

17. Taneva, S. G., Todorov, G., Petkanchin, I. B. \& Stoylov, S. P. (1987). Electrooptic study of the deionized form of bacteriorhodopsin. Eur. Biophys. J. 14, 415-421.

18. Mostafa, H. I. A., Varo, G., Toth-Boconadi, R. \& 
Keszthelyi, L. (1996). Electrooptical measurements on purple membrane containing bacteriorhodopsin mutants. Biophys. J. 70, 468-472.

19. Porschke, D. (1996). Electrostatics and electrodynamics of bacteriorhodopsin. Biophys. J. 71, 3381-3391.

20. Czege, J. \& Rheinisch, L. (1987). Light-scattering changes during the photocycle of bacteriorhodopsin. Acta Biochim. Biophys. Hung. 22, 463-478.

21. Czege, J. \& Rheinisch, L. (1991). The pH dependence of transient changes in the curvature of the purple membrane. Photochem. Photobiol. 54, 923-930. and references cited therein..

22. Porschke, D. (1996). Analysis of chemical and physical relaxation processes of polyelectrolytes by electric field pulse methods: a comparison of critical comments with facts. Ber. Bunsenges. Phys. Chem. 100, 715-720.

23. Porschke, D. (1985). Field induced interactions of phospholipid vesicles. Biochemistry, 24, 7981-7986.

24. Porschke, D., Meier, H. J. \& Ronnenberg, J. (1984). Interactions of nucleic acid double helices induced by electric field pulses. Biophys. Chem. 20, 225-235.

25. Varo, G. \& Lanyi, J. K. (1991). Kinetic and spectroscopic evidence for an irreversible step between deprotonation and reprotonation of the Schiff base in the bacteriorhodopsin cycle. Biochemistry, 30, 5008-5015.

26. Gergely, C., Zimanyi, L. \& Varo, G. (1997). Bacteriorhodopsin intermediate spectra determined over a wide $\mathrm{pH}$ range. J. Phys. Chem. B, 101, 9390-9395.

27. Zimanyi, L., Kulcsar, A., Lanyi, J. K., Sears, D. F., Jr \& Saltiel, J. (1999). Intermediate spectra and photocycle kinetics of the Asp96 $\rightarrow$ Asn mutant bacteriorhodopsin determined by singular value decomposition with self-modeling. Proc. Natl Acad. Sci. USA, 96, 4414-4419.

28. Shah, M. J. (1963). Electric birefringence of bentonite II. An extension of saturation birefringence theory. J. Phys. Chem. 67, 2215-2219.

29. Perrin, F. J. (1934). Mouvement brownien d'un ellipsoide (I). Dispersion dielectrique pour des molecules ellipsoidales. Physique, 5, 497-511.

30. Garcia de la Torre, J. \& Bloomfield, V. A. (1981). Hydrodynamic properties of complex, rigid, biological macromolecules: theory and application. Q. Rev. Biophys. 14, 81-139.

31. Antosiewic, J. \& Porschke, D. (1989). Volume correction for bead model simulations of rotational friction coefficients of macromolecules. J. Phys. Chem. 93, 5301-5305.

32. Wang, G. \& Porschke, D. (2003). Dipole reversal in bacteriorhodopsin and separation of dipole components. J. Phys. Chem. B, 107, 4632-4638.

33. Antosiewicz, J. \& Porschke, D. (1989). An unusual electrooptical effect observed for DNA fragments and its apparent relation to a permanent dipole moment associated with bent DNA. Biophys. Chem. 33, 19-30.

34. Heyn, M. P., Borucki, B. \& Otto, H. (2000). Chromophore reorientation during the photocycle of bacteriorhodopsin: experimental methods and functional significance. Biochim. Biophys. Acta, 1460, 60-74.

35. Luecke, H., Schobert, B., Richter, H. T., Cartailler, J. P. \& Lanyi, J. K. (1999). Structural changes in bacteriorhodopsin during ion transport at 2 Angstrom resolution. Science, 286, 255-260.

36. Schertler, G. F. X., Lozier, R., Michel, H. \& Oesterhelt, D. (1991). Chromophore motion during the bacteriorhodopsin photocycle: polarized absorption spectroscopy of bacteriorhodopsin and its M-state in bacteriorhodopsin crystals. EMBO J. 10, 2353-2361.

37. Heberle, J., Riesle, J., Thiedemann, G., Oesterhelt, D. \& Dencher, N. A. (1994). Proton migration along the surface and retarded surface to bulk transfer. Nature, 370, 379-382.

38. Alexiev, U., Mollaaghababa, R., Scherrer, P., Khorana, H. G. \& Heyn, M. P. (1995). Rapid long-range proton diffusion along the surface of the purple membrane and delayed proton transfer to the bulk. Proc. Natl Acad. Sci. USA, 92, 372-376.

39. Holz, M., Drachev, L. A., Mogi, T., Otto, H., Kaulen, A. D., Heyn, M. P. et al. (1989). Replacement of aspartic acid-96 by asparagines in bacteriorhodopsin slows both the decay of the $\mathrm{M}$ intermediate and the associated proton movement. Proc. Natl Acad. Sci. USA, 86, 2167-2171.

40. Porschke, D. (2002). Reaction coupling, acceptor $\mathrm{pK}$ and diffusion control in light induced proton release of bacteriorhodopsin. J. Phys. Chem. 106, 10233-10241.

41. Stoeckenius, W., Lozier, R. H. \& Bogomolni, R. A. (1979). Bacteriorhodopsin and the purple membrane of Halobacteria. Biochim. Biophys. Acta, 505, 215-278.

42. Subramaniam, S. \& Henderson, R. (2000). Molecular mechanism of vectorial proton translocation by bacteriorhodopsin. Nature, 406, 653-657.

43. Sass, H. J., Büldt, G., Gessenich, R., Hehn, D., Neff, D., Schlesinger, R. et al. (2000). Structural alterations for proton translocation in the M-state of wild-type bacteriorhodopsin. Nature, 406, 649-653.

44. Kühlbrandt, W. (2000). Bacteriorhodopsin-the movie. Nature, 406, 569-570.

45. Sachs, F. (2002). Retaining your identity under stress. Nature Struct. Biol. 9, 636-637.

46. Oesterhelt, D. \& Stoeckenius, W. (1974). Isolation of the cell membrane of Halobacterium halobium and its fraction in red and purple membrane. Methods Enzymol. 31, 667-670.

47. Rigler, R., Rabl, C. R. \& Jovin, T. M. (1974). A temperature-jump apparatus for fluorescence measurements. Rev. Sci. Instrum. 45, 580-588.

48. Humphrey, W., Dalke, A. \& Schulten, K. (1996). VMD-visual molecular dynamics. J. Mol. Graph. 14, $33-38$.

Edited by G. von Heijne 